Progress Performance Report

February 1, 1993
DOE/ER/40734--1

DE93 010070

\title{
Investigation of the Coherent Synchrotron Radiation Spectrum as a Probe of Charge Dynamics
}

\author{
A. J. Sievers \\ Laboratory of Atomic and Solid State Physics, Cornell University \\ and \\ Maury Tigner \\ $\min 26103$ \\ Laboratory of Nuclear Studies, Cornell University

\section{Executive Summary}

During the past funding period coherent synchrotron, Cerenkov and transition radiation have generated and measured in the far infrared spectral region. This represents the first identification and separation of the Cerenkov and transition radiation components with transition radiation producing the strongest signal. High intensity picosecond pulses of radiation are observed for all three sources. The angular dependence in the $\mathrm{mm}$ wave region is not the same as that given by high frequency radiation expressions since the wavelength and the beam radiator size are comparable so diffraction effects are important.

A prototype polarizing Michelson interferometer has been designed, constructed and is now being tested. The diffraction-limited design meets the demands of the rigorous space and experimental conditions in the linac area. The instrument is of rugged constructed using retroreflectors and hence is optically stable for long periods of time. It contains both reference and interferogram detectors to minimize the influence of linac drifts on the data analysis. To eliminate the intensity loss at low frequencies due to diffraction and waveguide effects the next generation of interferometer and detecior will have $2 x$ larger apernures 


\section{A Summary of Overall Progress}

A complete measurement of the coherent spectrum generated by a short charged particle bunch can be used to measure the spatial properties of the bunch itself, since its shape is proportional to the modulus squared of the Fourier transform of the longitudinal electron distribution. The first demonstration of a coherent spectrum was made with synchrotron radiation.[1] Measurements at the Cornell linac ratified this earlier measurement.[2] A key part of the current program is to measure the spectrum with sufficient precision so that the form factor of the bunch can be determined with a resolving power of at least 100 . In addition, since the output has a peak power of megawatts for picosecond pulse lengths, it can be used for time-resolved pump-probe experiments of hot plasmas in the spectral region where low-lying collective excitations are important.

Progress has been made on two different fronts during the past funding period.

\section{A. Identification of coherent radiation sources produced by electron bunches}

By combining room temperature Golay cell far $\mathbb{R}$ detectors with a number of cutoff filters, a qualitative study has been made of the different kinds of coherent radiation sources produced by electron bunches. Besides synchrotron we have also identified for the first time coherent transition and Cerenkov radiation generated by $\mathrm{mm}$ long electron bunches from the Cornell linac.

1. transition radiation

Transition radiation (TR) is produced by the passage of a charged particle through the interface between media with different dielectric constants[1]. It is caused by a collective response of the matter surrounding the particle trajectory to readjust to the electromagnetic field of the charged particle. Shown in Fig. 1(a) is a schematic picture corresponding to the geometry used in our experiment. A negatively charged particle is incident at $45^{\circ}$ with respect to a metallic mirror. It is easiest to visualize the transition radiation process by considering the nonrelativistic limit. In the low-frequency region where the metal acts like a near perfect conductor, its electric shielding effects can be represented in the nonrelativistic limit by an image charge placed at an equal distance behind the metal surface. As the charge approaches the boundary so does this image charge. The emitted radiation pattern with a frequency spectrum extending from the microwave to the $\mathrm{X}$-ray region [see Fig. $1(b)$ ] is emitted when the charged particle enters the metal and the charge and its image cancel.

For the Cornell linac the electrons are in the extreme relativistic limit $(\gamma=600)$ and the spectral radiation energy emitted by a single electron is

$$
E_{\omega} d \omega=\left(\frac{e^{2}}{\pi c}\right)\left[\ln \left(\frac{2}{1-\beta}\right)-1\right] d \omega
$$


where $E_{T}$ is the energy radiated per unit bandwidth. Note that the output is independent of frequency in this near mm wave region.

Our experiments have shown that the analysis must be modified if instead a charged particle bunch is incident on the conductor. If the bunch length is larger than the wavelength of the radiation, each charged particle in the bunch radiates independently and the intensity is proportional to the number of particles[e.g. Fig 1(b) at $10^{4} \mathrm{~cm}^{-1}$ ]; however, if the converse is true (e.g. at $10 \mathrm{~cm}^{-1}$ ), the bunch radiates coherently. Our experiments show that in the far IR the radiation intensity is proportional to the square of the number of electrons in the bunch just like for the earlier measurements of coherent synchrotron radiation[2]. For coherent TR the total energy per bunch in the far IR is measured to be $1.7 \mu \mathrm{J}$ for $5 \times 10^{9}$ electrons which is somewhat larger than obtained from synchrotron radiation. This TR value should be compared with that calculated for a $1-\mathrm{mm}$ Gaussian electron beam incident on a perfect conductor, namely $15 \mu \mathrm{J}$ for frequencies greater than $1 \mathrm{~cm}^{-1}$. The bunch length determines the spectral distribution of the far IR, as illustrated by the experimental data (solid dots) in Fig. 1(b).
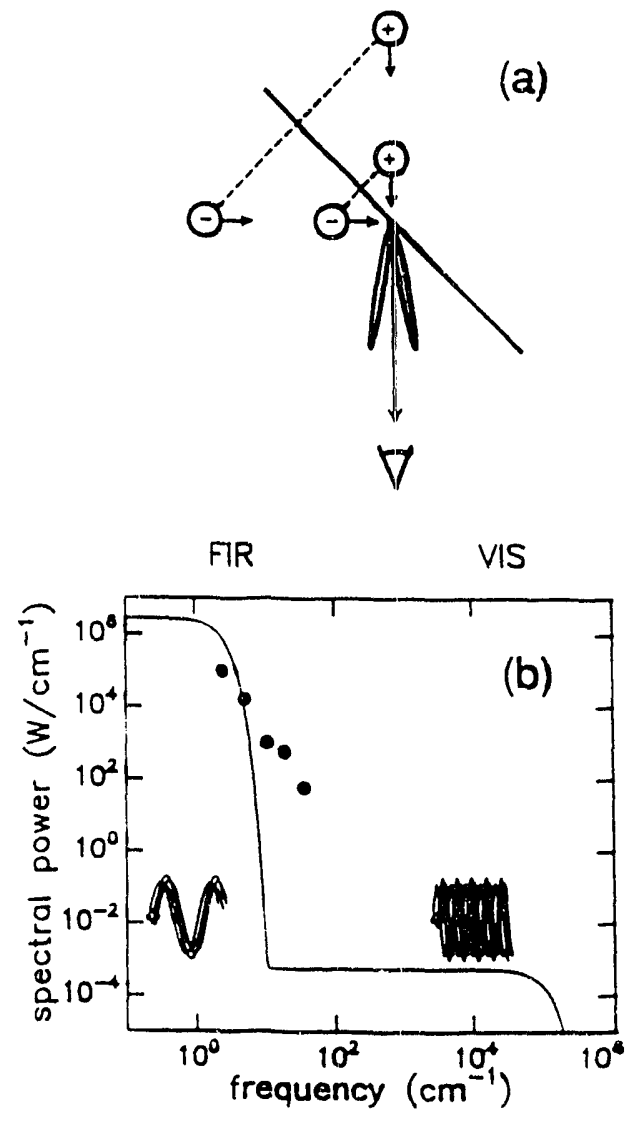

Figure 1 (a) Backward angular pattern of coherent TR for oblique incidence on a metal mirror. (b) Frequency spectra of transition radiation. Note the difference in spectral power levels between the coherent and incoherent regime. Solid dots: experimental data taken with the step filter interferometer. 


\section{Cerenkov radiation}

The first search for coherent Cerenkov radiation (CR) was made in the microwave region by Neighbours, et al. in 1987.[5] They observed radiation and reported that the Cerenkov angle is opened at long wavelengths. Four years later two groups in Japan reported coherent $\mathrm{CR}$ in the $\mathrm{mm}$ wave region.[6,7] In our study this past year, outlined below, we demonstrate that all of these investigators were observing coherent $\mathrm{TR}$ emitted from the metal vacuum window that the electron beam passed through and not coherent $\mathrm{CR}$ from the air path as they believed.

From our study of these two sources we expected that CR and TR would have similar angular and polarization dependences; however, one way to distinguish between them is by their relative strengths. The spectral energy of the $\mathrm{CR}$ emitted by a single electron passing through a dielectric with dielectric constant $\varepsilon$ is given by

$$
E_{C} d \omega=\left(\frac{e^{2} \omega \ell}{c^{2}}\right)\left(1-\frac{1}{\beta^{2} \varepsilon}\right) d \omega
$$

where $\ell$ is the path length of the electron in the medium. Note that the energy increases linearly with the emitted frequency, $\omega$. The ratio of the spectral energies between CR and TR is from Eqs. (1) and (2) then

$$
\frac{\mathrm{E}_{\mathrm{c}}}{\mathrm{E}_{\mathrm{T}}}=\left(\frac{\pi \omega \ell}{\mathrm{c}}\left[\frac{\left(1-\frac{1}{\varepsilon}\right)}{\ln \left(\frac{2}{1-\beta}\right)-1}\right]\right.
$$

Given that the dielectric constant of air $\varepsilon$ (air) $=1.00059$ and for a frequency $\omega=1 / \lambda=3 \mathrm{~cm}^{-1}$ so that coherent effects will prevail then for the parameter values of our experimental air chamber this ratio $\mathrm{E}_{\mathrm{C}} / \mathrm{E}_{\mathrm{T}}=0.006$. Clearly, $\mathrm{TR}$ can be investigated in air without a significant CR contribution.

In order to see coherent $\mathrm{CR}$ we filled a vacuum chamber with an atmosphere of $\mathrm{SF}_{6}$ gas which has a much larger dielectric constant than air, namely, $\varepsilon\left(\mathrm{SF}_{6}\right)=1.00207$. When the electron beam passed through this chamber we observed an $8 \%$ increase in the signal on the detector over that from the coherent TR for the vacuum case. Nearly the same increase was observed each time the chamber was pumped out and refilled again with this gas. We attribute this additional effect to coherent $\mathrm{CR}$ generated along the electron path. Equation (3) above gives a ratio for this configuration of $\mathrm{E}_{\mathrm{C}} / \mathrm{E}_{\mathrm{T}}=0.05$ in good agreement with experiment.

\section{B. Prototype Michelson interferometer design, construction and testing}

To measure the far in: ared spectrum of any of the coherent far infrared radiation sources puts constraints on the spectrometer. Of the three kinds that come to mind: prism, grating and interferometric, only the interferometer can readily cover a decade in frequency at high resolution. 


\section{Data analysis}

With this instrument the radiation is divided into two parts, one part is delayed with respect to the other and then the two parts are combined at the detector. The resultant interference pattern as a function of the distance of the delaying arm contains all the spectral information and is called an interferogram. For a single frequency $v=1 / \lambda$ the interferogram $\mathrm{g}(\mathrm{x})$ produced by the superposition of the two beams has a simple cosine form, namely,

$$
g(x)=2 S[1+\cos (2 \pi v x)]
$$

where $S$ is the intensity in one of the two equal intensity arms of the interferometer. If the source emits a continuous spectrum of frequencies $S(v)$ as is the case for the coherent spectrum of interest then

$$
g(x)=2 \int d v S(v)(1+\cos [2 \pi v x]) .
$$

Note that for $\mathrm{x}=0$ the total illumination is

$$
g(0) / 2=2 \int d v S(v)
$$

and the interferogram can be rearranged to give

$$
s(x)=g(x)-g(0) / 2=2 \int d v S(v)(\cos [2 \pi v x]) .
$$

The data now represent a cosine fourier transform of the spectrum of interest, $S(v)$. The inverse fourier transform gives the spectrum so that

$$
S(v)=\int d x s(x) \cos [2 \pi x v] .
$$

The longitudinal form factor of the bunch $S(z)$ is directly related to this spectrum with the following fourier transform,

$$
S(z)=\int d z[S(v)]^{\frac{1}{2}} \cos [2 \pi z v] .
$$

Because $S(v)$ enters Eq. (9) as the square root it is important that the spectrum be measured with good signal to noise in the high frequency region where the signal is small.

\section{Design}

The key component in a Michelson interferometer in the long wavelength regiopn is the beam spiitter. How can min waves be split 50 - 50 into two beams independent of the frequency? 
The best method is to use wire grid polarizers.[8] If the spacing between the wire grids is much less than the wavelength then the wave is reflected if the E-vector is directed along the wire direction and the wave is transmitted for the perpendicular polarization. By using $36 \mu \mathrm{m}$ tungsten wire on a coil winding machine, we have been able to construct 3 " diameter polarizers with $100 \mu \mathrm{m}$ wire spacing so that the polarization effect works at least down to $200 \mu \mathrm{m}$, a frequency of $50 \mathrm{~cm}^{-1}$.

For the 2.5 " prototype Michelson we arranged the polarizers as shown in Fig. (2) below. The unpolarized radiation enters the instrument from the left. One polarization is transmitted (shown) and the other is reflected (not shown). The transmitted linearly polarized radiation is no split equally by a second polarizer at $45^{\circ}$ with respect to the first one. This section is the Michelson interferometer. When the two amplitudes return through the polarizer they superimpose to form a linearly polarized beam but its orientation depends on the position of the movable mirror in the figure. Let's consider a single wavelength as an example. If the two mirrors have equal path difference then the returning radiation is vertically polarized and simply passes through the polarizer analyzer. However, if the moving mirror is shifted by $\lambda / 4$ with respect to the fixed mirror then the returning beam is horizontally polarized and is reflected to the detector by the polarizer analyzer. A cosine interferogram results with a black light position at equal paths.
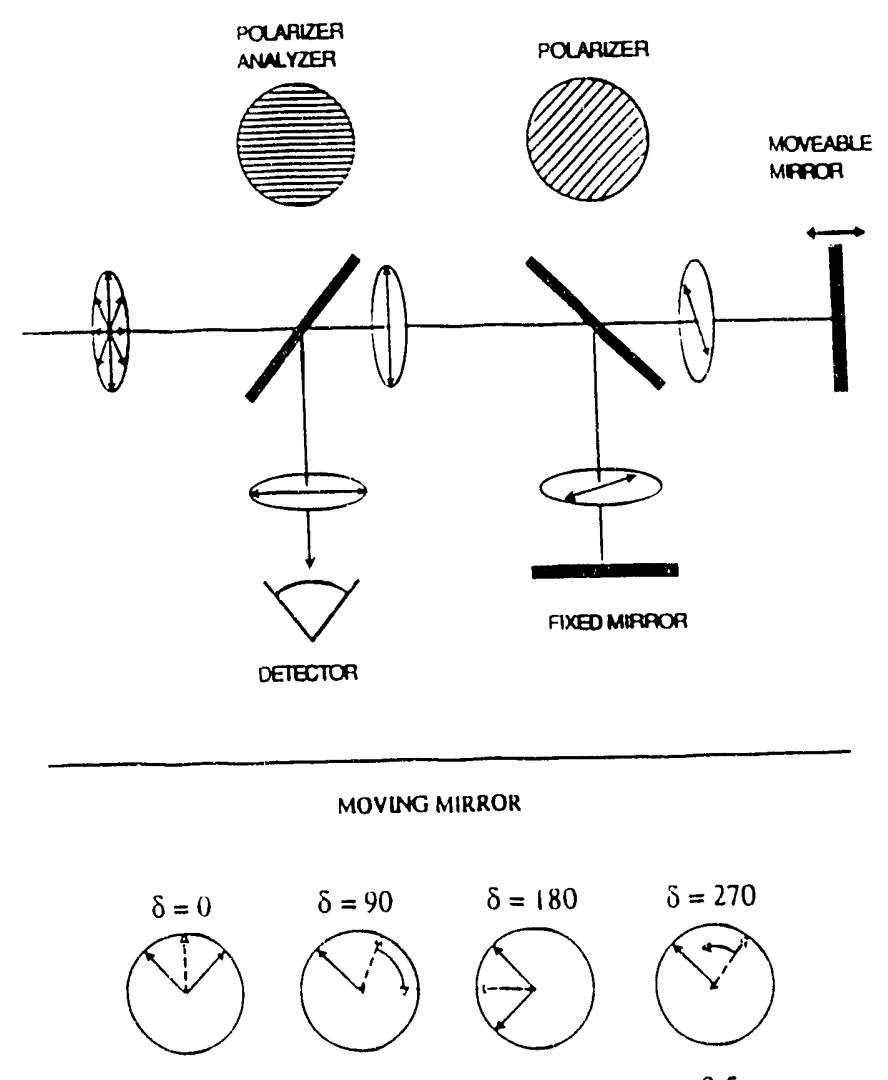

H. SIGNAL O

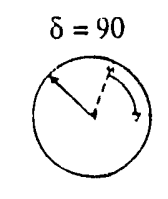

0.5

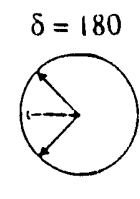

1.0

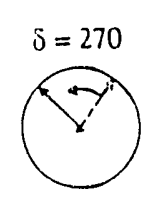

0.5

Figure 2. Schematic layout of the pclarizing Miicheison interferomeier. 
One of the practical advantages of this design besides the 50-50 beam splitting capability is that the first reflected beam from the polarized analyzer can be sent directly to a Golay cell detector and used as a reference signal to take out changes in the interferogram signal caused by drifts in the accelerator conditions during the time of the run.

\section{Tests}

To test the interferometer, we used a $\mathrm{Hg}$ arc source and a helium cooled detector to obtain an interferogram. The resultant interferogram versus optical path difference is shown in Fig. 3(a) below. The fourier transform was taken with a home-made program on a PC. A typical run takes 5 minutes. The intensity versus frequency results are shown in Fig. 3(b) below. The three strong absorption lines in the spectrum are due to the rotational spectrum of water vapor in the atmosphere. The 2.5 inch aperture of this instrument leads to loss of signal at long wavelengths because of diffraction effects. Additional tests with the Golay cell detectors show that the long wavelength radiation is suppressed even more by the detector itself. This occurs because of the long wavelength cutoff produced by the $3 \mathrm{~mm}$ aperture at the detector entrance. Hence wavelengths longer than $6 \mathrm{~mm}$ tend to be diffracted out of the beam path by the nearby optical edges as well as reflected rather than transmitted into the detector cavity.
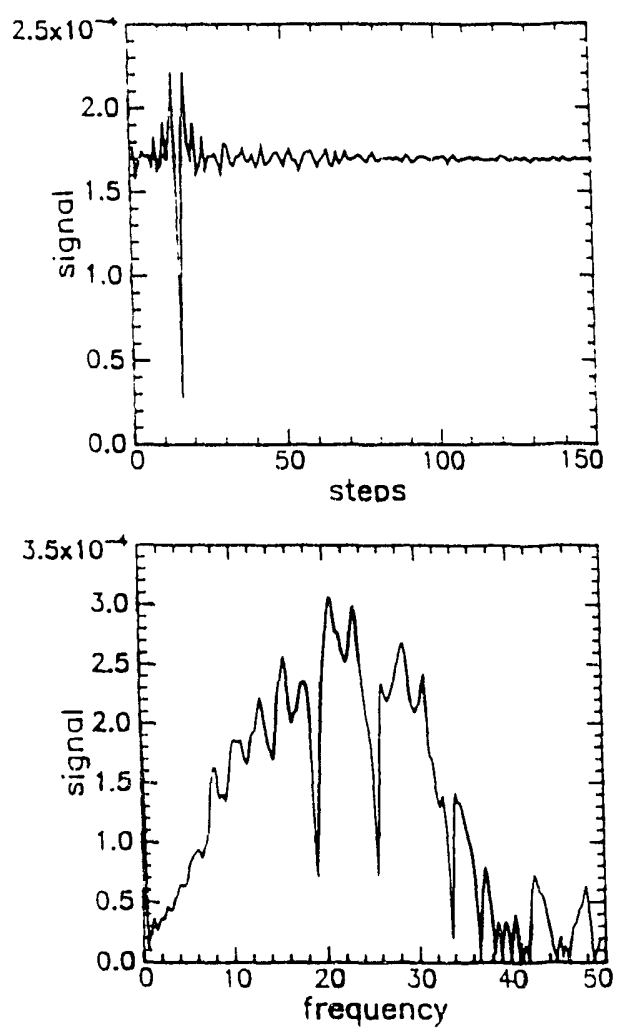

Figure 3. Interferometer test. (a) The black light interferogram versus optical path difference. (b) Far infrared spectrum versus frequency. 


\section{Problems and Favorable Developments}

The Golay cell detectors suppress the detection of long wavelength radiation because of the cutoff produced by the $3 \mathrm{~mm}$ aperture at the detector entrance. Hence wavelengths longer than $6 \mathrm{~mm}$ tend to be reflected rather than transmitted into the detector cavity. The prototype polarizing interferometer has been constructed and tested. It meets the demands of the experimentally confined situation but because of the small aperture size it too reduces the transmitted signal at long wavelengths because of diffraction effects at each optical surface. On the other hand, the instrument is portable, of rugged construction, contains a reference detector and is optically stable.

\section{Work to be Performed During the Succeeding Funding Period}

There are a number of tests and decisions that need to be made before starting construction of the full size polarizing Michelson interferometer.

\section{A. Finish testing the prototype grid interferometer to finalize interferometer} design.

There are two different kinds of interferometers that one can build for this spectral region. The white light or the black light machine. The identification is made when the optical path difference between the two arms is zero. We constructed the black light prototype machine so that we can make use of stable retroreflectors. On the other hand, it is easier to line up a white light machine because according to Eq. (6) one is tuning up the optical mirror alignment using the entire spectrum.

\section{B. How much remote control adjustment is required for these instrument?}

Because these instruments rely on optical interference to produce the desired effect some real time adjustment is required to tune up the instrument. Because of the high radiation activity in the experimental area this tuning must be done remotely. Since each remote control is expensive to install and one needs three of them for each optical component that needs adjusting, we must determine the minimum number required. Our first tests were made with no adjustments. Already we have found that some adjustment is required.

C. Determine the effect of the source position on interferometer spectrum.

Changing the lateral position of the electron bunch will change the effective position of the coherent source. An important experiment is to see how this shift changes the interference pattern at the detector and hence how this apodizes the frequency spectrum. If the effect is appreciable then the optical layout will have to be changed in such a way that the spot on the detector is less sensitive to small changes in the source position. 


\section{Purchase and test large aperture Golay cells at low frequencies.}

We have just discovered a new source of large aperture Golay detectors in Russia. We need to take delivery on two of these detectors and test their long wavelength response in the far infrared.

\section{E. Testing liquid helium cooled detectors.}

By using cooled InSb electron bolometer detectors it is possible in principle to increase the detector sensitivity by an additional factor of $10^{4}$. The question is whether or not these semiconducting detectors are also ultrasensitive to hard radiation. If they are insensitive to hard radiation then the next decision will be to decide between liquid helium cooling $(4.2 \mathrm{~K})$ versus mechanical Joule-Thompson cooling $(6 \mathrm{~K})$ for low temperature mm wave detectors. The mechanical cooling is more convenient but the mechanical vibrations may interfere with the detector sensitivity. If the InSb detectors are sensitive to hard radiation then one must turn to the slower composite bolometers to obtain enh nced sensitivity. These detectors are bulky and would influence the optical design of the machine.

\section{F. Construct large aperture low frequency grid interferometer.}

After the tests listed in items (a) through (e) are finished then we shall finish design and start construction of the large aperture polarizing interferometer.

\section{G. Search for coherent Bremsstràhlung radiation.}

In addition to the spectrometer building program described above we also intend to continue our qualitative investigation of the coherent sources produced by short electron bunches. Our plan is to look for coherent bremsstrahlung radiation. It is expected that the polarization and angular pattern will again be similar to that found for coherent $C R$ and TR but now the intensity at the detector will depend on the $Z^{2}$ of the target nuclei. The test will be to measure the radiation produced with the electron beam passing though $\mathrm{NaCl}$ compared to CsI, two materials with the same dielectric constant, so that the TR and CR will be the same, but with very different $Z$ so that the $B R$ will be different. Another compementry test will be to vary the bunch energy since the coherent $B R$ contribution depends linearly on the kinetic energy of the electron bunch while the TR and CR contributions show much weaker dependences.

\section{H. Test low temperature dispersive properties of KRS-5 prism material.}

We have discovered a possible low temperature prism material which may have sufficient dispersion in the $\mathrm{mm}$ wave region to be useful in a single shot spectrometer. If this material has the correct optical properties it may be possible to combine it with a sensitive detector array so that a spectrum of a single electron bunch can be measured. 


\section{References}

1. T. Nakazato et al., Phys. Rev. Lett. 63, 1245 (1989).

2. E. B. Blum, U. Happek and A. J. Sievers, Nucl. Instrum. Methods Phys. Res., Sect. A, 307, 568 (1991).

3. I. M. Frank and V. L. Ginzburg, J. Phys. (Moscow) 9, 353 (1945).

4. U. Happek, A. J. Sievers and E. B. Blum, Phys. Rev. Lett. 67, 2926 (1991).

5. J. R. Neighbours, et al., J. Appl. Phys. 61, 2741 (1987).

6. J. Ohkuma et al., Phys. Rev. Lett. 66, 1967 (1991).

7. Y. Shibata et al., Phys. Rev. A 44, 3449 (1991).

8. D. H. Martin, in Infrared and Millimeter Waves, Vol. 6, edited by K. Button, (Academic Press, 1982), Ch. 2. 

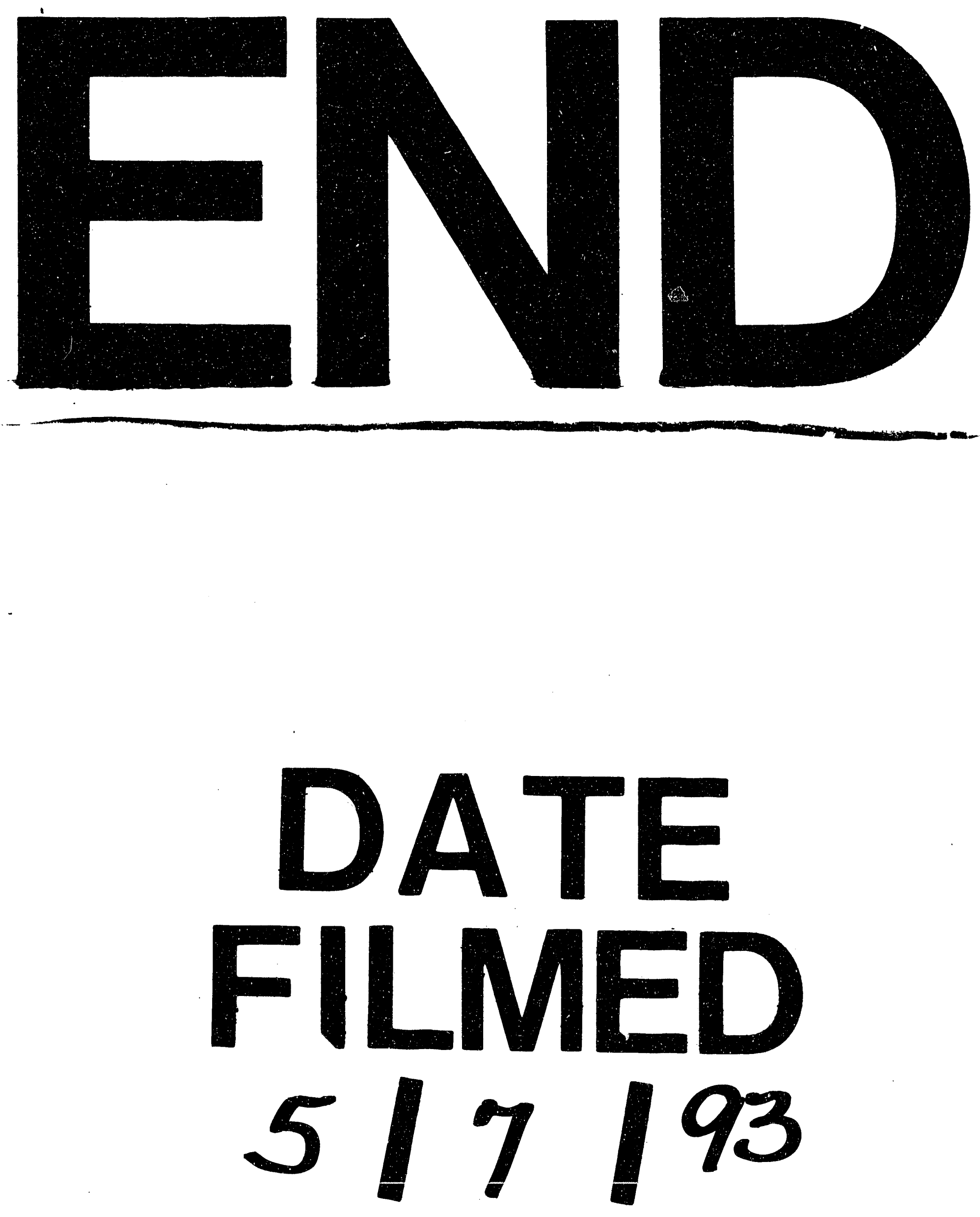

1 
